\title{
CIENCIAMATRIA
}

Revista Interdisciplinaria de Humanidades, Educación, Ciencia y Tecnología

Año VII. Vol. VII. N¹2. Enero - Junio. 2021

Hecho el depósito de ley: pp201602FA4721

ISSN-L: 2542-3029; ISSN: 2610-802X

Universidad Nacional Experimental Francisco de Miranda (UNEFM). Santa Ana de Coro. Venezuela

Bella Lourdes Argudo-Pesántez; Cecilia Ivonne Narváez-Zurita

Edwin Joselito Vásquez-Erazo

DOI $10.35381 / \mathrm{cm} \cdot \mathrm{v} 7 \mathrm{i} 12.440$

\section{Percepción de la Satisfacción del Cliente Externo: Centro Clínico Quirúrgico Ambulatorio Hospital del Día Azogues}

\section{Perception of External Customer Satisfaction: Outpatient Clinical Surgical Center Hospital del Día Azogues}

\author{
Bella Lourdes Argudo-Pesántez \\ bella.argudo.68@est.ucacue.edu.ec \\ Universidad Católica de Cuenca, Cuenca \\ Ecuador \\ https://orcid.org/0000-0002-3764-5545 \\ Cecilia Ivonne Narváez-Zurita \\ inarvaez@ucacue.edu.ec \\ Universidad Católica de Cuenca, Cuenca \\ Ecuador \\ https://orcid.org/0000-0002-7437-9880 \\ Edwin Joselito Vásquez-Erazo \\ evasqueze@ucacue.edu.ec \\ Universidad Católica de Cuenca, Cuenca \\ Ecuador \\ https://orcid.org/0000-0001-9817-6773
}

Recibido: 01 de octubre de 2020

Aprobado: 15 de diciembre de 2020 


\author{
CIENCIAMATRIA \\ Revista Interdisciplinaria de Humanidades, Educación, Ciencia y Tecnología \\ Año VII. Vol. VII. N¹2. Enero - Junio. 2021 \\ Hecho el depósito de ley: pp201602FA4721 \\ ISSN-L: 2542-3029; ISSN: 2610-802X \\ Universidad Nacional Experimental Francisco de Miranda (UNEFM). Santa Ana de Coro. Venezuela \\ Bella Lourdes Argudo-Pesántez; Cecilia Ivonne Narváez-Zurita \\ Edwin Joselito Vásquez-Erazo
}

\title{
RESUMEN
}

En salud la atención al paciente es lo prioritario, un servicio de calidad está relacionado con cubrir las expectativas del usuario e identificar los niveles de satisfacción del paciente. El objetivo de este estudio es evaluar la percepción de la satisfacción del cliente externo del servicio de consulta externa y diferentes prestaciones del CCQA Hospital del Día Azogues. Se aplicó el método descriptivo SERVQUAL, por medio de las cinco dimensiones de la calidad, con un diseño no experimental transversal, Se identificó las variables con mayor insatisfacción, en el tiempo de espera, medianamente satisfechos en la capacidad de respuesta en atender y resolver las inquietudes, aspectos con buenos resultados, como la presentación del personal, así como también el uso de protecciones de seguridad y la capacidad de los profesionales. En general los usuarios se sintieron satisfechos con el servicio, sin embargo, se identificaron aspectos débiles que requieren estrategias de mejoramiento.

Descriptores: Gestión; recursos humanos; competencias para la vida. (Palabras tomadas del Tesauro UNESCO).

\begin{abstract}
In health care, patient care is the priority. A quality service is related to meeting user expectations and identifying patient satisfaction levels. The aim of this study is to evaluate the perception of the satisfaction of the external customer of the outpatient service and different services of the CCQA Hospital del Día Azogues. The descriptive method SERVQUAL was applied, by means of the five dimensions of quality, with a nonexperimental transversal design. The variables with the greatest dissatisfaction were identified, in the waiting time, moderately satisfied in the capacity of response in attending and solving the concerns, aspects with good results, such as the presentation of the personnel, as well as the use of safety protections and the capacity of the professionals. In general, users were satisfied with the service; however, weak aspects were identified that require improvement strategies.
\end{abstract}

Descriptors: Management; human resources; life skills. (Words taken from the UNESCO Thesaurus). 


\section{CIENCIAMATRIA}

Revista Interdisciplinaria de Humanidades, Educación, Ciencia y Tecnología

Año VII. Vol. VII. N¹2. Enero - Junio. 2021

Hecho el depósito de ley: pp201602FA4721

ISSN-L: 2542-3029; ISSN: 2610-802X

Universidad Nacional Experimental Francisco de Miranda (UNEFM). Santa Ana de Coro. Venezuela

Bella Lourdes Argudo-Pesántez; Cecilia Ivonne Narváez-Zurita

Edwin Joselito Vásquez-Erazo

\section{INTRODUCCIÓN}

La calidad de los servicios de salud se ha venido estudiando en todos los países, de acuerdo con la Organización Mundial de la Salud (OMS), una atención de calidad se consigue cuando el paciente no tiene riesgo, y el servicio otorgado por los establecimientos de salud cubre sus expectativas. En salud la atención al paciente es lo prioritario, un servicio de calidad, está relacionado con las expectativas que tenga el usuario con respecto a la atención recibida, en este contexto la calidad no depende únicamente del profesional médico ni del equipamiento de última tecnología, sino también de la percepción y las expectativas que tiene el paciente del servicio (Guadalupe-Rivera, et al., 2019).

En la práctica de la salud se debe considerar principalmente el respeto para el usuario y el derecho que tenemos las personas a la protección de salud, los usuarios se vuelven muy exigentes para cubrir las expectativas, lo principal es mejorar la calidad de servicio, en el sector público donde los ciudadanos exigen buenos servicios, es difícil cumplir, en la realidad dentro del país los servicios públicos provocan insatisfacción, las instituciones públicas deben brindar una atención de calidad con calidez, conocer las inquietudes y con el interés de mejorar la calidad de salud pública, así como saber que prefiere el usuario, efectuar acciones para cubrir sus expectativas, con una orientación dirigida al usuario, requiere de un cambio de actitud (Sandoval, et al., 2018).

La calidad de la salud se puede conseguir cuando no existe riesgo para el paciente por

lo que la calidad depende de la confianza, ya sea en el profesional de salud en las diferentes especialidades y servicios, tecnologías de punta o atención personalizada. Para ofrecer los servicios de salud es necesario efectuar una permanente evaluación de los servicios que ofrecen los centros médicos públicos o privados, para incrementar la satisfacción de los clientes y de esta forma cubrir las expectativas del usuario.

Según Fitzpatrick, en sus propuestas, contiene 3 grandes principios: Que los profesionales de salud evalúen la satisfacción de los pacientes, a través de lo cual se 


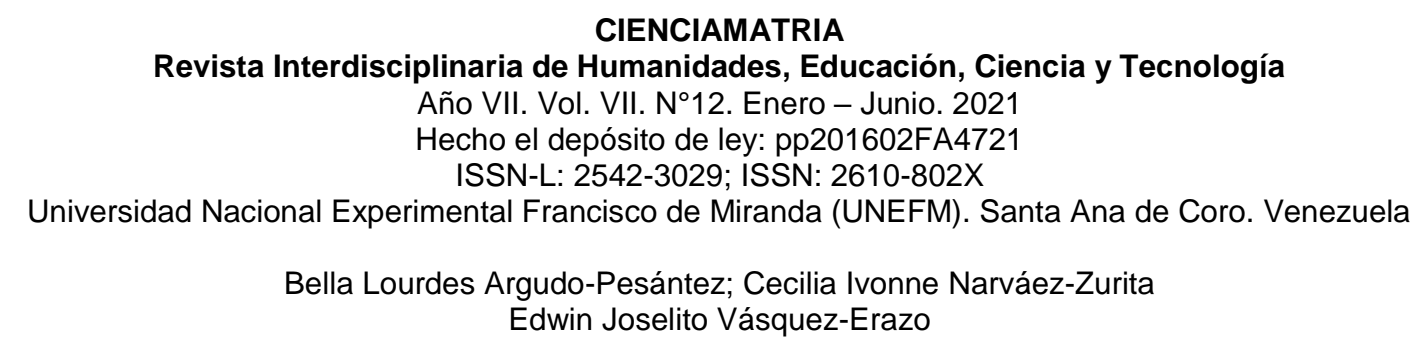

puede determinar el producto que es la calidad del servicio recibido o percibido en un establecimiento de salud, para de esta forma identificar las deficiencias para efectuar un mejoramiento en forma continua de los servicios de salud.

Para medir la calidad de la atención brindada a los pacientes en los establecimientos de salud, se debe utilizar procesos estandarizados, a través de indicadores que permitan identificar aspectos que tienen relación tanto con el personal de las unidades que brindan atención de salud, su capacidad, así como también el equipamiento y la comodidad de las instalaciones.

Identificar las inquietudes y percepciones de los pacientes y sus acompañantes que acuden a la consulta externa y otras prestaciones con las que cuenta los establecimientos de salud, en relación a la atención recibida y así poder conocer el nivel de satisfacción de los usuarios, para la elaboración de programas de mejoramiento continuo, cuyo fin principal es trazar tácticas para optimizar la calidad de estos servicios. (Ríos-Cáceres, et al., 2016)

En la rendición de cuentas en el año 2016, se presenta la Cobertura Institucional del CCQA Hospital del Dia Azogues, establecimiento de salud de segundo nivel de atención, que brinda los servicios a los siete Cantones de la Provincia de Cañar, y los 28 dispensarios del SSC, se atendieron a un total de 92.141 pacientes. (Romero, 2017). En el año 2018 el, CCQA Hospital del Día Azogues, ofreció 114.498, un número superior al 2016 y 2017, las más relevantes fueron endocrinología, con 10.000 atenciones y ginecología, con 9.000, las atenciones fueron mayores a las de años anteriores, se incrementaron los profesionales y equipo (Cañar, 2019).

Esta investigación tiene como objetivo, evaluar la percepción de la satisfacción del usuario de consulta externa y diferentes servicios que presta el CCQA Hospital del Día Azogues, identificar las inquietudes y percepciones de los pacientes para adoptar las mejores estrategias encaminadas a brindar un servicio de salud con calidad. 


\section{CIENCIAMATRIA}

Revista Interdisciplinaria de Humanidades, Educación, Ciencia y Tecnología

Año VII. Vol. VII. N¹2. Enero - Junio. 2021

Hecho el depósito de ley: pp201602FA4721

ISSN-L: 2542-3029; ISSN: 2610-802X

Universidad Nacional Experimental Francisco de Miranda (UNEFM). Santa Ana de Coro. Venezuela

Bella Lourdes Argudo-Pesántez; Cecilia Ivonne Narváez-Zurita

Edwin Joselito Vásquez-Erazo

\section{Referencial Teórico}

\section{Modelos de Satisfacción del Cliente externo en las organizaciones}

La implementación de un modelo de satisfacción del usuario, contribuye en optimizar la calidad del servicio en las empresas, a través de la percepción de los clientes por los servicios, permite además estudiar la personalidad, evaluar si el bien o servicio que prestan las empresas o entidades públicas o privadas, lograron cubrir sus expectativas, para ello es necesario tomar en cuenta; lo que les gusta, determinar la eficiencia o deficiencia, es subjetivo, entre los modelos están el tradicional, el persono lógico y el Kano, a continuación se presenta el diagrama de Kano (Carrillo, et al., 2016).

Diagrama dE rano

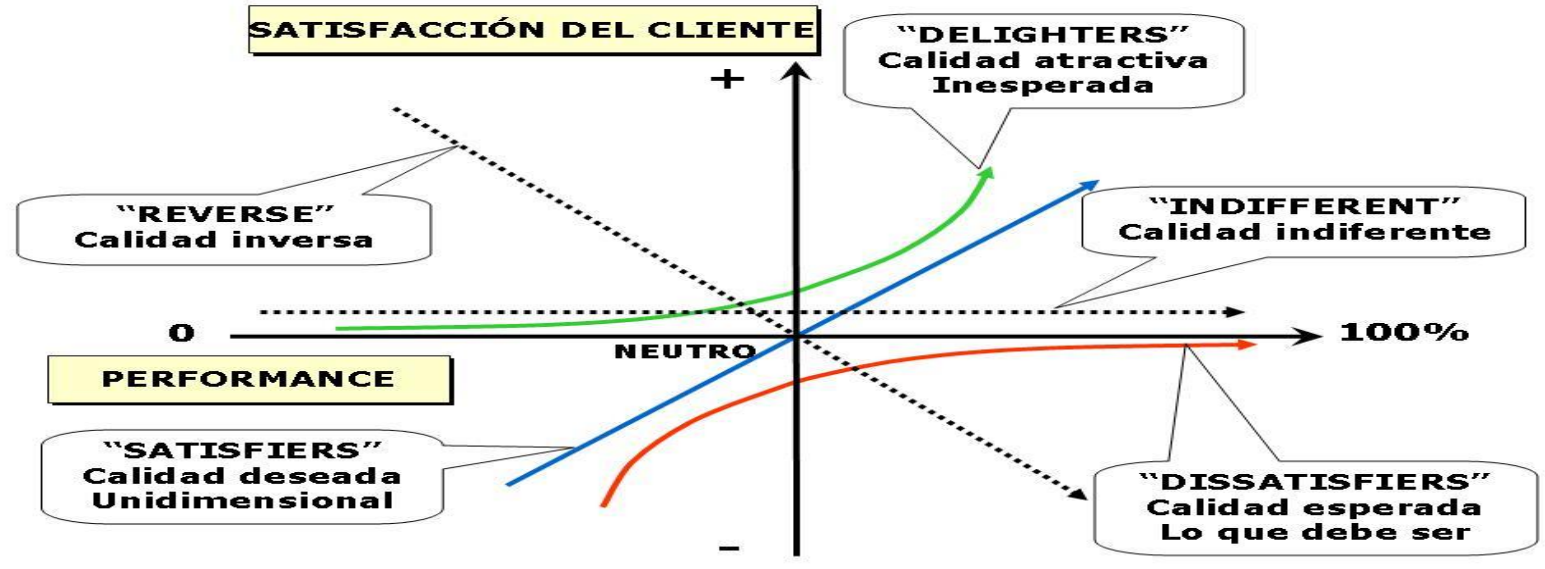

Figura 1. llustración 5: Modelo Kano.

Fuente: Carrillo, et al. (2016).

El modelo de satisfacción del cliente del profesor Noriaki Kano, es una teoría que se da a conocer a principios de los años 80 , perfeccionada por muchos años (30) por su creador, tiene relación con mejorar el producto o servicio, para de esta forma conseguir la satisfacción del cliente. La teoría del modelo Kano clasifica a las preferencias del usuario en 5 categorías: Calidad requerida, calidad atractiva, calidad unidimensional, 


\section{CIENCIAMATRIA \\ Revista Interdisciplinaria de Humanidades, Educación, Ciencia y Tecnología \\ Año VII. Vol. VII. N¹2. Enero - Junio. 2021 \\ Hecho el depósito de ley: pp201602FA4721 \\ ISSN-L: 2542-3029; ISSN: 2610-802X \\ Universidad Nacional Experimental Francisco de Miranda (UNEFM). Santa Ana de Coro. Venezuela \\ Bella Lourdes Argudo-Pesántez; Cecilia Ivonne Narváez-Zurita \\ Edwin Joselito Vásquez-Erazo}

calidad indiferente y calidad inversa. El modelo Kano se enfoca en las particularidades del servicio o producto (Pitaluga, 2015).

En la Revista Cubana de la Investigación Biomédica, varios autores expresan su criterio sobre la Gestión de la Innovación en los Servicios de Salud Pública, cuyo objetivo principal es el bienestar en la población, para lo que se debe efectuar un mejoramiento continuo, las unidades de salud pública tienen como propósito generar bienestar no lucro, por lo que la misión de los servicios de salud busca que las unidades y centros de salud, puedan brindar un buen servicio que logre satisfacer los requerimientos de los usuarios, tomando en cuenta la creatividad e innovación, brindando un servicio de salud de calidad. (Teran, et al., 2017).

\section{Calidad en el servicio en las Organizaciones}

La calidad del servicio tiene relación directa con la satisfacción del cliente, por lo tanto, evaluar esta relación será de gran ayuda en la toma de decisiones dentro de la administración de un servicio (Mora-Contreras, 2011), y servirá como una estrategia para potenciar la estructura y organización funcional, tomando en cuenta las necesidades y deseos de los potenciales clientes o usuarios, permitiendo de esta manera aumentar la competitividad del servicio o producto ofrecido (Mendez-Matovelle, et al., 2020).

La fidelidad y satisfacción del usuario son elementos imprescindibles para aumentar la competitividad de las instituciones, ya que permitirá mantener una relación a largo plazo con los clientes actuales; mismos que atraerán nuevos clientes potenciales que harán crecer los ingresos y promoción de la institución (González-Arias, et al., 2015). 


\section{CIENCIAMATRIA}

Revista Interdisciplinaria de Humanidades, Educación, Ciencia y Tecnología

Año VII. Vol. VII. N¹2. Enero - Junio. 2021

Hecho el depósito de ley: pp201602FA4721

ISSN-L: 2542-3029; ISSN: 2610-802X

Universidad Nacional Experimental Francisco de Miranda (UNEFM). Santa Ana de Coro. Venezuela

Bella Lourdes Argudo-Pesántez; Cecilia Ivonne Narváez-Zurita

Edwin Joselito Vásquez-Erazo

\section{Trilogía de Juran}

Joseph Juran propone que debe existir una planificación, control y mejoramiento de la calidad; (Terán, 2020).

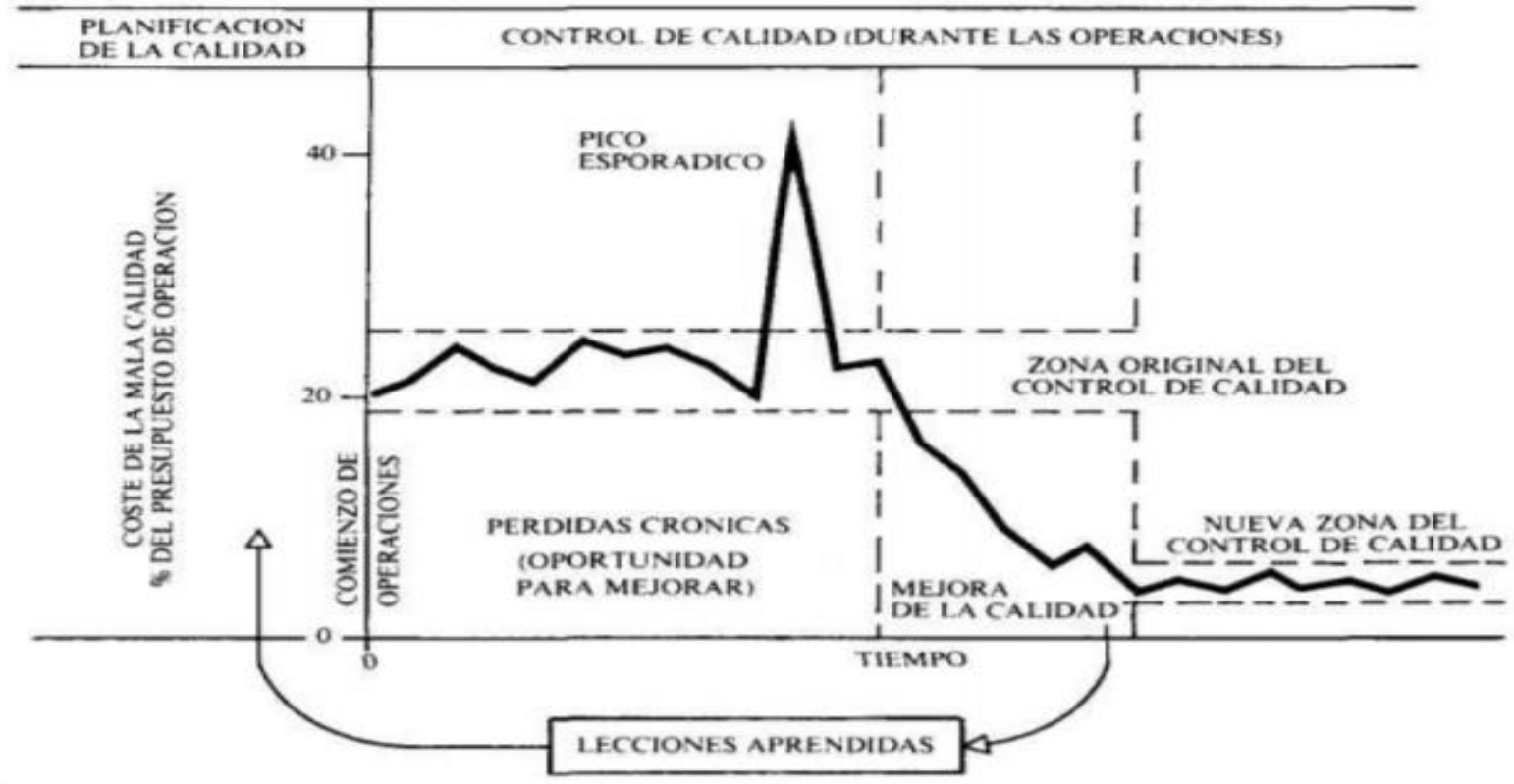

Figura 2. Trilogía de Juran.

Fuente: Juran (1988).

La Salud y calidad, dentro de la atención en salud la calidad es un factor muy importante; ya que consiste dar una respuesta y trato adecuado a las necesidades del usuario. Brindado un tratamiento efectivo, eficiente, de calidad, y de acuerdo con la patología que el usuario presente (Terán, 2020). La organización mundial de la salud (OMS) ha incorporado a la eficacia de atención como un objetivo en salud para toda la población, ya que manifiesta que todas las personas tienen derecho a recibir el más alto nivel de salud. 


\section{CIENCIAMATRIA \\ Revista Interdisciplinaria de Humanidades, Educación, Ciencia y Tecnología \\ Año VII. Vol. VII. N¹2. Enero - Junio. 2021 \\ Hecho el depósito de ley: pp201602FA4721 \\ ISSN-L: 2542-3029; ISSN: 2610-802X \\ Universidad Nacional Experimental Francisco de Miranda (UNEFM). Santa Ana de Coro. Venezuela \\ Bella Lourdes Argudo-Pesántez; Cecilia Ivonne Narváez-Zurita \\ Edwin Joselito Vásquez-Erazo}

\section{Calidad de atención en salud.}

En 1980 Donabedian, defina a la atención de calidad como aquella que busca propagar el bienestar del usuario, tomando en consideración las ganancias y las pérdidas.

Donabedian ha establecido dos principios de calidad

- Calidad: Habilidad para efectuar de manera correcta lo que le corresponde y de esta manera satisfacer las necesidades de aquellos a los que se brinde un servicio.

- Calidez: Es un valor del ser humano, que se define como preocupación sincera por el bienestar de otra persona que acude a una institución.

Dimensiones de la calidad.

- Personal: Trato del equipo de salud con los usuarios,

- Técnica: Hace referencia a la calidad de las técnicas e instrumentos utilizados; tomando en cuenta el manejo y minimización de riesgos,

- Ambiental: Condiciones físicas del entorno y la comodidad brindada al paciente Atributos necesarios en los servicios de salud:

- Eficiencia: Utilizar de buena manera los recursos para cumplir con los objetivos planificados,

- Efectividad: El grado de beneficio que se puede generar con los recursos disponibles en la población tratada

- Eficacia: Obtener resultados óptimos

- Fiabilidad: Atender al paciente correctamente desde el momento de su recepción, para generar una buena imagen inmediatamente

- Rapidez: Realizar la atención medica dentro de un tiempo aceptable para el usuario tratado. Se recomienda evitar que el usuario permanezca mucho tiempo en sala de espera

- Cortesía: El personal de salud deberá tratar a sus pacientes con respeto, amabilidad y educación 


\section{CIENCIAMATRIA}

Revista Interdisciplinaria de Humanidades, Educación, Ciencia y Tecnología

Año VII. Vol. VII. N¹2. Enero - Junio. 2021

Hecho el depósito de ley: pp201602FA4721

ISSN-L: 2542-3029; ISSN: 2610-802X

Universidad Nacional Experimental Francisco de Miranda (UNEFM). Santa Ana de Coro. Venezuela

Bella Lourdes Argudo-Pesántez; Cecilia Ivonne Narváez-Zurita

Edwin Joselito Vásquez-Erazo

- Seguridad: El usuario no debe dudar, ni sentir miedo o riesgo al momento de ser atendido

- Accesibilidad: Facilidad con la que cuenta el usuario para acceder al servicio cuando desee

- Comunicación: Comunicarnos de manera clara y sencilla con el usuario, evitando un vocabulario técnico que lo pueda confundir (Terán, 2020).

En el artículo "Satisfacción de los usuarios hospitalizados en el servicio de medicina interna, con el cuidado de enfermería se determinó que los usuarios que recibieron atención en Medicina Interna en la ciudad de Xalapa, Veracruz, México; se encontraban satisfechos con la atención brindada. Las variables utilizadas para el estudio fueron: cuidados de enfermería dentro de los cuales se tomaban las actitudes de los profesionales de enfermería con sus usuarios como: amabilidad, comprensión, confianza, respeto, paciencia, etc. El instrumento que se usó para calificar la atención fue el "Patient Satisfaction Instrument (PSI)"; mismo que cuenta con 3 dimensiones: cuidado técnico profesional, confianza y educación al paciente (Blázquez \& Quezada, 2014).

En el estudio de Infantes-Gómez en el 2017 llamado "Calidad de atención y grado de satisfacción de los usuarios de consulta externa del Centro de Atención de Medicina Complementaria del Hospital III lquitos-2016"; el objetivo fue evaluar los factores asociados a la calidad del servicio en medicina en el Hospital III lquitos; estudio en el que se utilizó la escala SERVQUAL modificada para calcular el nivel de satisfacción de los pacientes; y además se analizaron factores asociados como: sexo, edad, nivel de educación y condición del paciente. Obteniendo como resultados una satisfacción general de: $72,3 \%$ en capacidad de respuesta, $73,7 \%$ de empatía, $77,1 \%$ de seguridad y 73,6 de elementos tangibles. Además, en cuentos a los factores asociados: el sexo y la edad se asociaron con todas dimensiones analizadas (Infantes-Gómez, 2017). 


\section{CIENCIAMATRIA \\ Revista Interdisciplinaria de Humanidades, Educación, Ciencia y Tecnología \\ Año VII. Vol. VII. N¹2. Enero - Junio. 2021 \\ Hecho el depósito de ley: pp201602FA4721 \\ ISSN-L: 2542-3029; ISSN: 2610-802X \\ Universidad Nacional Experimental Francisco de Miranda (UNEFM). Santa Ana de Coro. Venezuela \\ Bella Lourdes Argudo-Pesántez; Cecilia Ivonne Narváez-Zurita \\ Edwin Joselito Vásquez-Erazo}

En el estudio "Nivel de satisfacción de usuarios hospitalizados en servicio de cirugía del Hospital Regional Lambayeque - 2018"; se buscó el nivel de satisfacción de 255 usuarios hospitalizados en esta institución, utilizando el instrumento SERVQUAL; gracias al cual, se pudieron obtener los siguientes resultados: $76,1 \%$ en servicio recibido; $83,02 \%$ en aspectos tangibles, con menor porcentaje se encuentra la dimensión "capacidad de respuesta" en la que únicamente se obtuvo el 66,09\%; y en último lugar con 53,88\% se encontraban "los trámites para las altas" (Sandoval, et al., 2018).

Los modelos multidimensionales han servido de basa para evaluar la relación entre calidad y satisfacción desde un punto de vista estratégico; que permitirán poner en marcha programas funcionales para mejorar el rendimiento de la empresa o institución. (Mora-Contreras, 2011).

\section{Modelo de Escala multidimensional SERVQUAL}

Creado por Parasuraman, Zethaml y Berry, mismo que tiene como objetivo conocer la percepción del usuario al utilizar el servicio prestado. (Numpaque-Pacabaque, et al., 2016). En este modelo los usuarios califican el servicio prestado por una entidad pública o privada, donde las variables cualitativas pasan a convertirse en sumas numéricas y con porcentjes. (Valencia, 2015)

Dimensiones de la escala multidimensional SERVQUAL:

De acuerdo con el estudio de Valencia; "Aplicación del modelo SERVQUAL para la medición de la calidad del servicio de mantenimiento y limpieza de establecimientos educativos en la empresa SYRY"; se menciona que las cinco dimensiones que se deben aplicar para medir la calidad de los servicios:

- Confiabilidad: Habilidad

- de brindar un servicio como se ofertó al cliente.

- Responsabilidad: servicio rápido y oportuno. 


\section{CIENCIAMATRIA}

Revista Interdisciplinaria de Humanidades, Educación, Ciencia y Tecnología

Año VII. Vol. VII. N¹2. Enero - Junio. 2021

Hecho el depósito de ley: pp201602FA4721

ISSN-L: 2542-3029; ISSN: 2610-802X

Universidad Nacional Experimental Francisco de Miranda (UNEFM). Santa Ana de Coro. Venezuela

Bella Lourdes Argudo-Pesántez; Cecilia Ivonne Narváez-Zurita

Edwin Joselito Vásquez-Erazo

- Seguridad: Los empleados están capacitados para atender con amabilidad y cortesía, brindando confianza al usuario.

- Empatía: Brindar atención personalizada a los clientes.

- Bienes tangibles: Relacionado con el aspecto y equipamiento de las instalaciones. En el estudio realizado por Huerta y Angela en enero de 2010, se aplicó la metodología SERVQUAL de alta confiabilidad, a los pacientes de emergencia del Hospital de Lima, para ello se aplicó esta metodología, dentro de este estudio, se pudo determinar que el paciente está satisfecho en el indicador principal, para de esta forma evaluar los parámetros de atención recibida por el paciente, aquí se analiza varios aspectos personales y contextuales del usuario, a través de encuestas se conocen información que ayuda a los directivos de la entidad de salud a identificar cuáles son las falencias, para de esta manera mejorar los servicios y cubrir las expectativas de los usuarios, beneficiando tanto a la entidad como a los pacientes (Huerta-Medina, 2015).

Varios investigadores realizaron el estudio a la atención que recibieron en dicho centro, a través de las dimensiones de: tangibilidad, fiabilidad, capacidad de respuesta, seguridad y empatía, con los resultados del estudio se puedo determinar que no existía diferencias ni a favor ni en contra. (Guadalupe-Rivera, et al. 2019).

En otro estudio realizado a 384 usuarios que acudieron a establecimiento de saluda indicado anteriormente sobre percepción de calidad y fiabilidad de sus servicios utilizando la escala SERVQUAL; dentro del cual se analizaron factores como: tiempo de espera para el servicio, horarios, solución de problemas y confianza transmitida (Fariño, et al., 2018).

En la investigación realizada por Arbeláez y Mendoza en los Centros de Salud en el Ecuador, a través de la herramienta SERVQUAL se pudo conocer las expectativas y percepciones del usuario en el servicio de salud, determinándose que la satisfacción del usuario se da por la confianza que brinda el personal Médico y la deficiencia se produce 


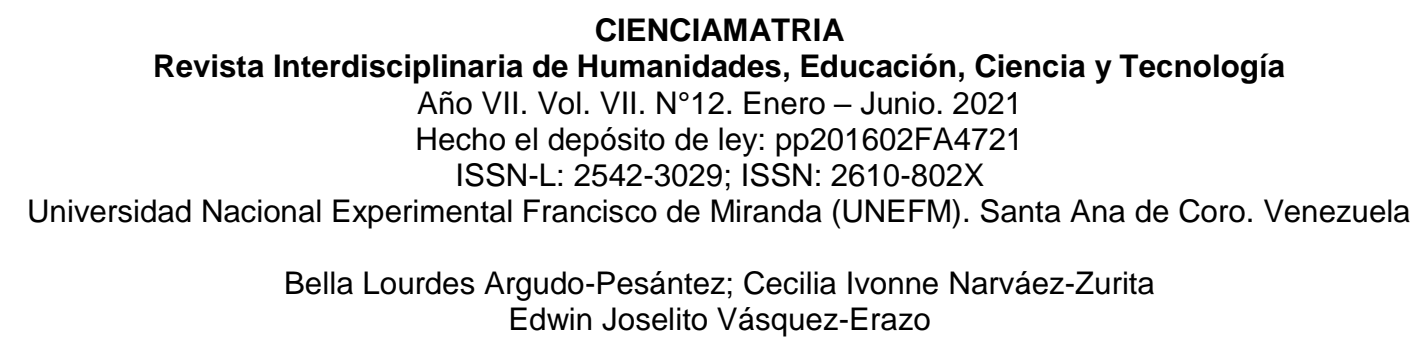

por la demora en la atención, dilatación en el agendamiento de citas, falta de insumos y recursos, en los establecimientos de salud pública (Arbeláez \& Mendoza, 2017).

En su artículo de investigación Desarrollo del Modelo SERVQUAL para la medición de la calidad del servicio en la empresa de publicidad Ayuda Experto, la investigadora expresa que, los servicios son actividades identificables e intangibles, permite identificar el porcentaje de buena atención y las necesidades del paciente, se debe analizar además sus aspectos cuantitativos y cualitativos; en forma adicional permite conocer factores importantes de los usuarios o clientes, que no se puede ver, recoge las expectativas y percepciones de los clientes, permite realizar comparaciones con otras similares, por lo que para medir la calidad del servicio agrupa cinco dimensiones: Fiabilidad, Sensibilidad, Seguridad, Empatía y Elementos tangibles (Matsumoto, 2014).

\section{Modelo SERVQHOS}

Herramienta que permite medir los aspectos objetivos y subjetivos relacionados con a la estructura y la calidad de atención en hospitalaria (Numpaque-Pacabaque \& RochaBuelvas, 2016), con el fin de obtener los resultados de la calidad de servicio con menos elementos que el SERVQUAL.- En un artículo de salud varios investigadores aplicaron la metodología SERVQHOS y se pudo establecer los aspectos, de atención recibida, los cuales producían mayor satisfacción a los pacientes atendidos en la consulta externa de un establecimiento hospitalario público de México, los resultados obtenidos mostraron que tanto la atención brindada por los médicos a sus pacientes, así como la agilidad en los trámites para la atención, horarios convenientes y tiempos de espera tenían una buena calificación (Godínez, et al., 2014).

Modelo SERVPERF el cual fue propuesto por Cronin y Taylor en 1992, hace relación a la percepción del usuario por los servicios recibidos (Service Performance), cuenta con los mismos elementos del SERVQUAL y únicamente se diferencia ya que este modelo 


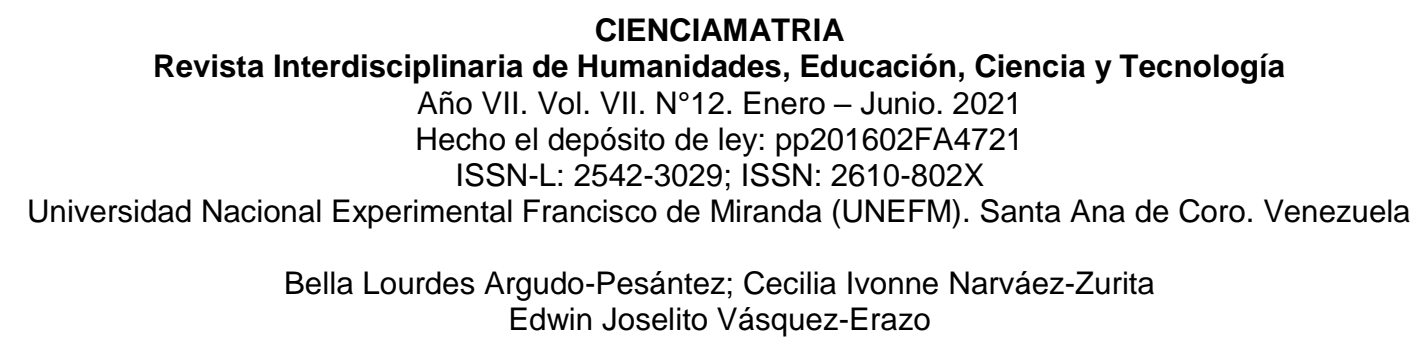

no considera dentro de la evaluación las expectativas de los clientes (Ibarra \& Casas, 2015).

En el estudio realizado por algunos autores relacionado con la forma en la que perciben a calidad de la atención médica brindada a los usuarios a la escala SERVQUAL y a la SERVPERF, se presenta una explicación de la correlación que existe entre la calidad del servicio, la satisfacción del consumidor y fines de comprar o adquisición de un bien o servicio, concluyéndose en que la satisfacción del usuario es contar con un producto de la calidad (Ibarra \& Casas, 2015).

En el estudio realizado en el año 2019 por Jacqueline Sánchez et al llamado "Implementación del modelo SERVPERF como herramienta para el diagnóstico y propuesta de mejora en la prestación del Servicio. Caso: Instituto Ecuatoriano de Seguridad Social Agencia Riobamba"; se tiene como objetivo utilizar el instrumento SERVPERF para delimitar las falencias de este Centro e implementar mejoras de acuerdo con las necesidades de los afiliados a este centro. Se pudo evidenciar que existen deficiencias en el servicio de esta institución; presentadas por mal funcionamiento en talento humano, falta de capacitación, deficiencias en la rapidez de atención y ausencia de empatía. (Sánchez-Lunavictoria, et al., 2019).

\section{MÉTODO}

La metodología de la presente investigación se sustenta desde un tipo descriptivo, a través de esta metodología se intenta estar al tanto de las percepciones, se podrá describir la calidad de atención médica del establecimiento, por medio de las cinco dimensiones de la calidad, con diseño no experimental transversal, debido a que la recopilación de la información y descripción se realizó en un tiempo establecido, siendo la población de análisis los pacientes que acudieron a la consulta externa de las diferentes especialidades y servicios del CCQA Hospital del Día Azogues. Se empleó la 


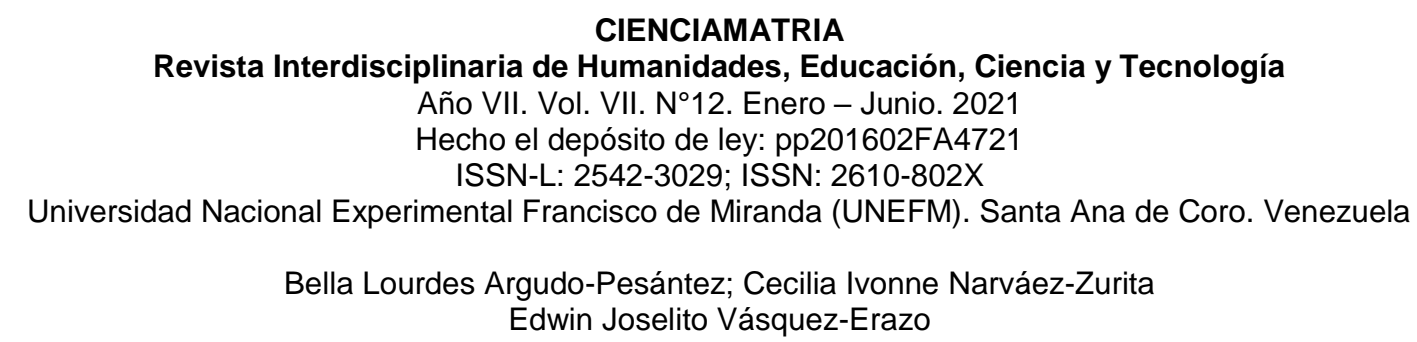

encuesta y cuestionario online para la recopilación de información, mientras que su procesamiento se realizó a través de estadística descriptiva.

En este estudio tuvo alcance cuantitativo, un diseño metodológico que utilizó un cuestionario modelo SERVQUAL incluye 5 dimensiones, la tangibilidad, fiabilidad, capacidad de respuesta, seguridad y empatía.

\section{Universo de estudio y tratamiento muestral}

Se efectuó un muestreo por conveniencia, que es una técnica no probabilística, elaborada de manera aleatoria, formado por los pacientes que acudieron a la consulta externa de las diferentes especialidades y servicios que presta el CCQA Hospital del Día Azogues que para este estudio fueron 80 pacientes.

\section{RESULTADOS}

En el estudio realizado, el cuestionario de la encuesta efectuada estuvo formado por 10 preguntas, mediante las cuales se pudo medir las dimensiones de tangibilidad, factibilidad, capacidad de respuesta, seguridad y empatía, respecto a la percepción que tienen los pacientes del servicio brindado en el CCQA Hospital del Dia Azogues, obteniendo los siguientes resultados:

En cuanto a las deficiencias encontradas, la única pertenece a la dimensión de fiabilidad, en lo que tiene que ver con los tiempos de espera con un $29 \%$, y cercanos a la media están la dimensión de empatía en lo que tiene que ver con la comprensión frente a sus necesidades y emociones del paciente y la capacidad de respuesta a los trámites que realiza la entidad de salud en un 43 y $40 \%$.

Aspectos con buenos resultados, se identificaron como la presentación del personal con el porcentaje de $63 \%$, así como también el uso de protecciones de seguridad y la capacidad de los profesionales en un $61 \%$. 


\section{CIENCIAMATRIA}

Revista Interdisciplinaria de Humanidades, Educación, Ciencia y Tecnología

Año VII. Vol. VII. N¹2. Enero - Junio. 2021

Hecho el depósito de ley: pp201602FA4721

ISSN-L: 2542-3029; ISSN: 2610-802X

Universidad Nacional Experimental Francisco de Miranda (UNEFM). Santa Ana de Coro. Venezuela

Bella Lourdes Argudo-Pesántez; Cecilia Ivonne Narváez-Zurita

Edwin Joselito Vásquez-Erazo

En general los usuarios se sintieron satisfechos con el servicio, sin embargo, se identificaron debilidades que requieren estrategias de mejoramiento.

Estas preguntas reflejan que el servicio de atención de trámites, agendamiento de citas médicas no es completamente satisfactoria, por lo que los servidores no atienden completamente las necesidades del paciente. Se debe controlar y elevar la calidad de atención tanto en la consulta externa y diferentes servicios del establecimiento de salud. Las dimensiones con mayor porcentaje de satisfacción de los usuarios, fuero las dimensiones de tangibilidad y seguridad.

\section{PROPUESTA}

La propuesta para el análisis de la percepción de la satisfacción del cliente externo del CCQA Hospital del Dia Azogues, en su esquema, tiene como objetivo identificar las dificultades que tienen los pacientes, cuando acuden a recibir atención en el establecimiento de salud objeto de estudio, para lo cual se propone el esquema de cuatro etapas en forma secuencial, en donde se detalla el contenido de cada una de ellas como se muestra a continuación.

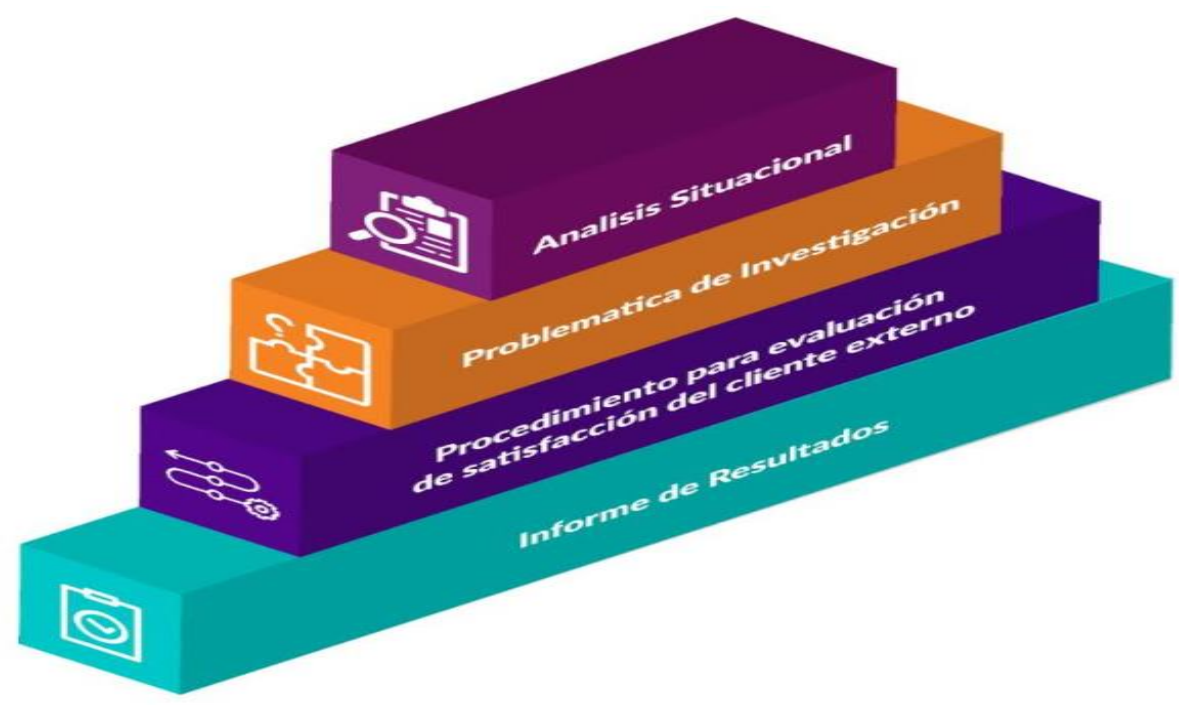

Figura 3. Esquema de la propuesta. 


\section{CIENCIAMATRIA}

Revista Interdisciplinaria de Humanidades, Educación, Ciencia y Tecnología

Año VII. Vol. VII. N¹2. Enero - Junio. 2021

Hecho el depósito de ley: pp201602FA4721

ISSN-L: 2542-3029; ISSN: 2610-802X

Universidad Nacional Experimental Francisco de Miranda (UNEFM). Santa Ana de Coro. Venezuela

Bella Lourdes Argudo-Pesántez; Cecilia Ivonne Narváez-Zurita

Edwin Joselito Vásquez-Erazo

\section{Análisis Situacional.}

La presente investigación se realiza en base a los resultados de la encuesta efectuada a los pacientes que acuden a recibir atención en el CCQA Hospital del Día Azogues, el cuestionario constó de 10 preguntas, en donde se pudo identificar de acuerdo con el cuestionario Modelo SERVQUAL, que muestra la complacencia de los pacientes y la calidad de la atención, a través del diagnóstico resumido en dimensiones:

A través del estudio de los resultados encontrados que tienen relación con los indicadores de las 5 dimensiones, permitieron establecer las dificultades y errores que tiene que ver con la percepción de la satisfacción del usuario externo, ante el servicio recibido, a continuación, se presenta el procedimiento de acuerdo con el cuestionario que muestran la satisfacción de los pacientes y la calidad del servicio por dimensión de acuerdo con la percepción de la calidad del servicio:

\section{Dimensión de Tangibilidad}

\section{Tabla 1.}

CCQA Hospital del Día Azogues.

\begin{tabular}{|c|c|c|c|c|c|c|}
\hline Indicadores & Satisfechas & $\begin{array}{l}\text { Medianamente } \\
\text { Satisfechos \% }\end{array}$ & Insatisfechas & Vacías & Total & $\%$ \\
\hline $\begin{array}{l}\text { ¿Cómo calificaría la presentación } \\
\text { del personal del establecimiento? }\end{array}$ & $63 \%$ & $30 \%$ & $5 \%$ & $3 \%$ & 80 & $100 \%$ \\
\hline $\begin{array}{l}\text { ¿Considera usted que las } \\
\text { instalaciones } \\
\text { (equipamiento e infraestructura) } \\
\text { del establecimientor son } \\
\text { apropiadas? }\end{array}$ & $54 \%$ & $40.00 \%$ & $4 \%$ & $2 \%$ & 80 & $100 \%$ \\
\hline $\begin{array}{l}\text { ¿Considera que tanto las sillas, } \\
\text { camillas y ambientes fueron } \\
\text { confortables para su atención? }\end{array}$ & $51 \%$ & $43 \%$ & $4 \%$ & $2 \%$ & 80 & $100 \%$ \\
\hline
\end{tabular}

Fuente: Tomado de encuestas. 


\author{
CIENCIAMATRIA \\ Revista Interdisciplinaria de Humanidades, Educación, Ciencia y Tecnología \\ Año VII. Vol. VII. N¹2. Enero - Junio. 2021 \\ Hecho el depósito de ley: pp201602FA4721 \\ ISSN-L: 2542-3029; ISSN: 2610-802X \\ Universidad Nacional Experimental Francisco de Miranda (UNEFM). Santa Ana de Coro. Venezuela \\ Bella Lourdes Argudo-Pesántez; Cecilia Ivonne Narváez-Zurita \\ Edwin Joselito Vásquez-Erazo
}

\title{
Análisis e interpretación
}

Esta dimensión se compone de indicadores como la presentación del personal del establecimiento, la infraestructura, equipamiento, camillas y sillas del CCQA Hospital del Día en es prácticamente nuevo tiene 7 años de creación, por lo que el usuario tiene donde esperar de forma cómoda, lo que hace que el usuario tenga una buena percepción del establecimiento objeto de estudio.

En lo relacionado a la presentación del personal, las instalaciones comodidad de camillas, sillas y ambientes confortables, el resultado de las encuestas muestra que: El $63 \%$ de pacientes manifiestan estar satisfechos, el 30\% medianamente satisfechos, el $5 \%$ se encuentran insatisfechos con esta variable y el $2 \%$ no contestaron.

De los 80 usuarios encuestados, expresan que están satisfechos el 54\% por que el establecimiento es nuevo y adecuado, así como con también con su equipamiento, el $40 \%$ expresan estar medianamente satisfechos, el $4 \%$ insatisfechos y el $2 \%$ no contestan.

El $51 \%$ de encuestados manifiesta satisfacción por la cantidad de camillas, sillas y ambientes apropiados de los servicios de la entidad de salud, el $43 \%$ se encuentran medianamente satisfechos, el $4 \%$ insatisfechos y un $2 \%$ no contestan. 


\section{CIENCIAMATRIA}

Revista Interdisciplinaria de Humanidades, Educación, Ciencia y Tecnología

Año VII. Vol. VII. N¹2. Enero - Junio. 2021

Hecho el depósito de ley: pp201602FA4721

ISSN-L: 2542-3029; ISSN: 2610-802X

Universidad Nacional Experimental Francisco de Miranda (UNEFM). Santa Ana de Coro. Venezuela

Bella Lourdes Argudo-Pesántez; Cecilia Ivonne Narváez-Zurita

Edwin Joselito Vásquez-Erazo

\section{Dimensión de Fiabilidad}

Tabla 2.

CCQA Hospital del Día Azogues.

\begin{tabular}{lcccccc}
\hline \multicolumn{1}{c}{ Indicadores } & Satisfechas & $\begin{array}{c}\text { Medianament } \\
\text { e Satisfechos }\end{array}$ & Insatisfechas & Vacías & Total & $\%$ \\
\hline $\begin{array}{l}\text { ¿Considera que los } \\
\text { tempos de espera para } \\
\text { la atención, están de } \\
\text { acuerdo con sus } \\
\text { expectativas? }\end{array}$ & $29 \%$ & $46 \%$ & $23 \%$ & $2 \%$ & 80 & $\begin{array}{c}100 \\
\%\end{array}$ \\
\hline $\begin{array}{l}\text { ¿Considera que los } \\
\text { horarios establecidos en } \\
\text { la institución de salud } \\
\text { son apropiados? }\end{array}$ & $56 \%$ & $31 \%$ & $11 \%$ & $2.00 \%$ & 80 & 100 \\
\hline
\end{tabular}

Fuente: Tomado de encuestas

\section{Análisis e interpretación}

El trabajo que se realiza en relación con el tiempo de espera del usuario para recibir la atención es la primera dimensión de los elementos de la fiabilidad, esperar mucho tiempo antes de ser atendidos produciría inconvenientes en el servicio, lo correcto sería de 0 a 25 minutos.

Evaluar la satisfacción de los pacientes del establecimiento en estudio, permitió conocer

el trabajo que se realiza en relación con el tiempo de espera del usuario para recibir la atención.

Estos indicadores se calcularon en base a las respuestas de los pacientes, donde únicamente el $29 \%$ están satisfechos con los tiempos lo que es relativamente bajo, el $46 \%$ expresan estar medianamente satisfechos, un $23 \%$ de ellos, manifiestan estar insatisfecho con el tiempo de espera para el servicio y el $2 \%$ no contesta. 


\section{CIENCIAMATRIA}

Revista Interdisciplinaria de Humanidades, Educación, Ciencia y Tecnología

Año VII. Vol. VII. N¹2. Enero - Junio. 2021

Hecho el depósito de ley: pp201602FA4721

ISSN-L: 2542-3029; ISSN: 2610-802X

Universidad Nacional Experimental Francisco de Miranda (UNEFM). Santa Ana de Coro. Venezuela

Bella Lourdes Argudo-Pesántez; Cecilia Ivonne Narváez-Zurita

Edwin Joselito Vásquez-Erazo

En lo que respecta a los horarios establecidos, los pacientes están medianamente satisfechos en $56 \%$, el $31 \%$ se encuentran medianamente satisfechos, el $11 \%$ están insatisfecho con este indicador, y el $2 \%$ no contestan.

\section{Dimensión Capacidad de Respuesta}

Tabla 3.

CCQA Hospital del Día Azogues.
Indicadores
Satisfechas
Medianamente
Insatisfechas Vacías Total

¿Cómo considera la atención del personal de salud para resolver

$40.00 \%$

$39 \%$

$18 \%$

$3 \%$

$80 \quad 100 \%$ inquietudes y reclamos?

Fuente: Tomado de encuestas.

\section{Análisis e interpretación.}

En los establecimientos de salud es muy importante, escuchar y resolver las quejas, lo cual no sucede, por lo que existe insatisfacción del paciente; por esta razón debe existir el compromiso de los servidores y profesionales de salud por brindar a los usuarios un servicio de calidad para evitar quejas y reclamos y cubrir sus expectativas.

En lo que tiene que ver con que los empleados atendieron y resolvieron las inquietudes y reclamos, el $40 \%$ de los usuarios manifiesta estar satisfechos, el $39 \%$ medianamente satisfechos, el 18\% se encuentran insatisfechos y $3 \%$ no contestan, lo cual demuestra que la satisfacción está por debajo de la media, lo cual representa una alerta que se debe tomar en cuenta. 


\section{CIENCIAMATRIA}

Revista Interdisciplinaria de Humanidades, Educación, Ciencia y Tecnología

Año VII. Vol. VII. N¹2. Enero - Junio. 2021

Hecho el depósito de ley: pp201602FA4721

ISSN-L: 2542-3029; ISSN: 2610-802X

Universidad Nacional Experimental Francisco de Miranda (UNEFM). Santa Ana de Coro. Venezuela

Bella Lourdes Argudo-Pesántez; Cecilia Ivonne Narváez-Zurita

Edwin Joselito Vásquez-Erazo

\section{Dimensión de Seguridad}

\section{Tabla 4.}

CCQA Hospital del Día Azogues.

\begin{tabular}{|c|c|c|c|c|c|c|}
\hline Indicadores & Satisfechas & $\begin{array}{r}\text { Medianamente } \\
\text { Satisfechos }\end{array}$ & Insatisfechas & Vacías & Total & $\%$ \\
\hline $\begin{array}{lcc}\text { ¿Cómo calificaría } & \text { la } \\
\text { capacidad } & \text { de } & \text { los } \\
\text { Profesionales de Salud? }\end{array}$ & $61 \%$ & $33 \%$ & $3 \%$ & $3 \%$ & 80 & 100 \\
\hline $\begin{array}{lr}\text { 10. ¿Considera que las } \\
\text { protecciones de seguridad } \\
\text { del personal } \\
\text { establecimiento } \\
\text { apropiadas? }\end{array}$ & $61 \%$ & $33 \%$ & $3 \%$ & $3 \%$ & 80 & 100 \\
\hline
\end{tabular}

Fuente: Tomado de encuestas.

\section{Análisis e interpretación.}

En esta dimensión se evalúa el servicio, aptitudes y competencias de los servidores y profesionales de la salud. Con la finalidad de conocer la percepción de la calidad de servicio, los conocimientos, capacidades y habilidades de los profesionales de salud del establecimiento, al momento de prestar un servicio, los usuarios expresaron su criterio que está por encima de la media, por lo que de acuerdo al porcentaje $61 \%$ se sienten seguros al recibir la atención de los médicos, enfermeras y auxiliares, están satisfechos con este indicador, el $33 \%$ medianamente satisfechos, seguido del $3 \%$ que no lo está y un $3 \%$ que no contestan, en lo que corresponde a las protecciones de seguridad del personal, de igual manera los usuarios muestran $61 \%$ de satisfacción, el $33 \%$ medianamente satisfechos, seguido del $3 \%$ que no lo está y un $3 \%$ que no contesta. 


\section{CIENCIAMATRIA}

Revista Interdisciplinaria de Humanidades, Educación, Ciencia y Tecnología

Año VII. Vol. VII. N¹2. Enero - Junio. 2021

Hecho el depósito de ley: pp201602FA4721

ISSN-L: 2542-3029; ISSN: 2610-802X

Universidad Nacional Experimental Francisco de Miranda (UNEFM). Santa Ana de Coro. Venezuela

Bella Lourdes Argudo-Pesántez; Cecilia Ivonne Narváez-Zurita

Edwin Joselito Vásquez-Erazo

\section{Dimensión de Empatía.}

\section{Tabla 5.}

CCQA Hospital del Día Azogues.

\begin{tabular}{|c|c|c|c|c|c|c|}
\hline Indicadores & Satisfechas & $\begin{array}{r}\text { Medianamente } \\
\text { Satisfechos }\end{array}$ & Insatisfechas & Vacías & Total & $\%$ \\
\hline $\begin{array}{l}\text { ¿Considera que le } \\
\text { brindaron confianza en la } \\
\text { atención tanto los } \\
\text { Médicos, Enfermeras y } \\
\text { Auxiliares? }\end{array}$ & $60 \%$ & $31 \%$ & $6 \%$ & $3 \%$ & 80 & 100 \\
\hline $\begin{array}{l}\text { ¿Existió comprensión } \\
\text { frente a sus necesidades y } \\
\text { emociones? }\end{array}$ & $44 \%$ & $50 \%$ & $4 \%$ & $6 \%$ & 80 & 100 \\
\hline
\end{tabular}

Fuente: Tomado de encuestas.

\section{Análisis e interpretación.}

En esta esta etapa se evalúa la empatía de todo el equipo de salud, de las respuestas de esta pregunta, se pudo identificar, que el $60 \%$ de satisfacción, los pacientes respondieron que el personal de médicos, enfermeras y auxiliares, es decir el equipo de salud, brindan una atención media alta, el 31\% estuvieron medianamente satisfechos, el $6 \%$ insatisfechos y el $3 \%$ no contestaron, en la pregunta de que si existió comprensión frente a sus necesidades y emociones, el $44 \%$ de los encuestados estuvieron satisfechos, el $55 \%$ medianamente satisfechos, el $4 \%$ se encontraron insatisfechos con los servicios y el $6 \%$ no contestan. 


\section{CIENCIAMATRIA \\ Revista Interdisciplinaria de Humanidades, Educación, Ciencia y Tecnología \\ Año VII. Vol. VII. N¹2. Enero - Junio. 2021 \\ Hecho el depósito de ley: pp201602FA4721 \\ ISSN-L: 2542-3029; ISSN: 2610-802X \\ Universidad Nacional Experimental Francisco de Miranda (UNEFM). Santa Ana de Coro. Venezuela \\ Bella Lourdes Argudo-Pesántez; Cecilia Ivonne Narváez-Zurita \\ Edwin Joselito Vásquez-Erazo}

\section{Problema de Investigación}

En vista de la importancia que tiene la atención en salud, dependiendo de la percepción de la satisfacción del cliente y la humanización de las prestaciones de salud, se hace necesario identificar la percepción que tiene el paciente sobre el servicio recibido, una vez evaluado el servicio se puede determinar los problemas existentes en la actualidad, para dar solución se debe tomar las medidas para cambiar los aspectos que tuvieron mala calificación, con el afán de mejorar el nivel de satisfacción de los pacientes que acuden a recibir atención en el establecimiento de salud objeto de estudio.

En cuanto a las deficiencias que se encontraron en la evaluación efectuada en estudio se pudo determinar que pertenece a la dimensión de fiabilidad, en lo que tiene que ver el bajo porcentaje de calificación que tienen los tiempos de espera con un porcentaje del $29 \%$, y la dimensión de capacidad de respuesta a los trámites que realiza la entidad de salud en un $40 \%$.

Se puede evidenciar que existen problemas de eficacia del servicio, ya que los funcionarios de la entidad objeto de estudio, se demoran en la atención de trámites y citas médicas, por esta razón los pacientes están medianamente satisfechos ya que no atienden sus necesidades en forma oportuna.

Para dar solución a estas deficiencias se debe revisar las políticas y protocolos de la institución, identificar el problema y plantear soluciones; así como mantener y reforzar los porcentajes de satisfacción de los usuarios en las dimensiones de tangibilidad y seguridad, que fueron las de mayor satisfacción.

Para Mejorar los tiempos de espera de los afiliados se establecerá un cronograma de atenciones, el cual se controlará, través del personal de chalecos rojos con supervisión del jefe administrativo de la entidad.

En lo que tiene que ver con el bajo porcentaje de disposición de ayudar de parte de los funcionarios, en la atención de trámites y retraso de agendamiento de citas, se 


\section{CIENCIAMATRIA}

Revista Interdisciplinaria de Humanidades, Educación, Ciencia y Tecnología

Año VII. Vol. VII. N¹2. Enero - Junio. 2021

Hecho el depósito de ley: pp201602FA4721

ISSN-L: 2542-3029; ISSN: 2610-802X

Universidad Nacional Experimental Francisco de Miranda (UNEFM). Santa Ana de Coro. Venezuela

Bella Lourdes Argudo-Pesántez; Cecilia Ivonne Narváez-Zurita

Edwin Joselito Vásquez-Erazo

efectuarán capacitaciones al personal de trabajo social, admisión y área administrativa, para que todo trámite que ingrese tenga la atención en el menor tiempo.

Para lo cual se debe utilizar un formato, en el que el personal defina la capacitación que se requiera y de esta forma mejorar la percepción del servicio que tienen los usuarios del establecimiento en estudio, como se explica en la siguiente tabla.

\section{Tabla 6.}

Cronograma de Capacitaciones.

\begin{tabular}{|c|c|c|c|c|}
\hline Ciudad: & $\begin{array}{l}\text { CCQA HOSPTIAI } \\
\text { Azogues }\end{array}$ & $\begin{array}{l}\text { DEL DIA } \\
\text { Fecha } \\
\text { (dd/mm/ } \\
\text { aaaa): }\end{array}$ & $\begin{array}{l}\text { AZOGUES } \\
1 / 12 / 2020\end{array}$ & \\
\hline \multicolumn{5}{|c|}{$\begin{array}{l}\text { Nombres y Apellidos del Servidor o } \\
\text { Servidora }\end{array}$} \\
\hline \multicolumn{5}{|c|}{ Responsable de Capacitación } \\
\hline No. & $\begin{array}{c}\text { Temas Referenciales Para } \\
\text { Capacitarse }\end{array}$ & $\begin{array}{c}\text { Respon } \\
\text { sable de } \\
\text { la } \\
\text { Capacit } \\
\text { ación } \\
\text { Tema }\end{array}$ & $\begin{array}{c}\text { Fecha de } \\
\text { Inducción del } \\
\text { Tema }\end{array}$ & $\begin{array}{l}\text { Duración de la } \\
\text { Inducción } \\
\text { (hh:mm) }\end{array}$ \\
\hline 1 & Servicios de la Institución & & & \\
\hline 2 & Código de Ética & & & \\
\hline 3 & Disponibilidad de servicio & & & \\
\hline 4 & $\begin{array}{l}\text { Reglamento de Seguridad y } \\
\text { Salud }\end{array}$ & & & \\
\hline 5 & Obligaciones del personal & & & \\
\hline 6 & Disponibilidad de servicio & & & \\
\hline 7 & $\begin{array}{l}\text { Acceso asignación de citas } \\
\text { médicas Web y ventanilla }\end{array}$ & & & \\
\hline
\end{tabular}

Fuente: Los autores. 


\section{CIENCIAMATRIA \\ Revista Interdisciplinaria de Humanidades, Educación, Ciencia y Tecnología \\ Año VII. Vol. VII. N¹2. Enero - Junio. 2021 \\ Hecho el depósito de ley: pp201602FA4721 \\ ISSN-L: 2542-3029; ISSN: 2610-802X \\ Universidad Nacional Experimental Francisco de Miranda (UNEFM). Santa Ana de Coro. Venezuela \\ Bella Lourdes Argudo-Pesántez; Cecilia Ivonne Narváez-Zurita \\ Edwin Joselito Vásquez-Erazo}

\section{Procedimiento para Evaluación de Satisfacción del Cliente Externo}

En el presente estudio, se procedió a evaluar la percepción de satisfacción del cliente externo, en el CCQA Hospital del Día Azogues, por lo que es de gran importancia evaluar los servicios ofrecidos, con el fin de identificar fortalezas y debilidades que permitan desarrollar estrategias en búsqueda de mejorar el servicio.

A través del estudio de los resultados encontrados que tienen relación con los indicadores de las 5 dimensiones, permitieron establecer las dificultades y errores que tiene que ver con la percepción de la satisfacción del usuario externo,

El análisis comienza determinando con que herramienta de evaluación lo realizaremos, en este estudio utilizamos un cuestionario Modelo SERVQUAL, que muestra los porcentajes de satisfacción por la calidad de la atención recibida por parte de los usuarios, a través del diagnóstico resumido en dimensiones de: tangibilidad, fiabilidad, capacidad de respuesta, seguridad y empatía, además se puede identificar y aplicar las operaciones correctivas o preventivas correspondientes, por lo que es de suma importancia elaborar estrategias que permitan mejor los servicios de salud.

\section{Tabla 7.}

PROCEDIMIENTO DE EVALUACIÓN DE SATISFACCIÓN A USUARIOS

\section{Objetivo}

Determinar la percepción de la satisfacción del cliente externo del

Centro Clínico Quirúrgico Hospital del Dia Azogues

\section{Alcance}

Este procedimiento inicia al determinar el instrumento de evaluación, y finaliza al aplicar las acciones correctivas o preventivas correspondientes. 


\section{CIENCIAMATRIA}

Revista Interdisciplinaria de Humanidades, Educación, Ciencia y Tecnología

Año VII. Vol. VII. N¹2. Enero - Junio. 2021

Hecho el depósito de ley: pp201602FA4721

ISSN-L: 2542-3029; ISSN: 2610-802X

Universidad Nacional Experimental Francisco de Miranda (UNEFM). Santa Ana de Coro. Venezuela

Bella Lourdes Argudo-Pesántez; Cecilia Ivonne Narváez-Zurita

Edwin Joselito Vásquez-Erazo

Dimensione

\begin{tabular}{|c|c|c|c|}
\hline $\mathrm{S}$ & \begin{tabular}{ll}
\multicolumn{2}{c}{ Preguntas } \\
¿Cómo calificaría la \\
presentación & del \\
personal & del \\
establecimiento? & \\
\end{tabular} & frecuencia & Valor cualitativo \\
\hline & $\begin{array}{l}\text { ¿Considera usted que } \\
\text { las instalaciones físicas } \\
\text { (equipamiento } \\
\text { infraestructura) } \\
\text { establecimiento del } \\
\text { apropiadas? }\end{array}$ & $54 \%$ & Satisfactorio \\
\hline Tangibilidad & $\begin{array}{l}\text { ¿Considera } \text { que tanto } \\
\text { las sillas, camillas y } \\
\text { ambientes r fueron } \\
\text { confortables para su } \\
\text { atención? }\end{array}$ & $51 \%$ & Satisfactorio \\
\hline \multirow[b]{2}{*}{ Fiabilidad } & $\begin{array}{l}\text { ¿Considera que los } \\
\text { tiempos de espera para } \\
\text { la atención, están de } \\
\text { acuerdo con sus } \\
\text { expectativas? }\end{array}$ & $29 \%$ & Insatisfactorio \\
\hline & $\begin{array}{l}\text { ¿Considera que los } \\
\text { horarios establecidos en } \\
\text { la institución de salud } \\
\text { son apropiados? }\end{array}$ & $56 \%$ & Satisfactorio \\
\hline $\begin{array}{l}\text { Capacidad } \\
\text { de } \\
\text { Respuesta }\end{array}$ & $\begin{array}{l}\text { ¿Cómo considera la } \\
\text { atención del personal de } \\
\text { salud para resolver } \\
\text { inquietudes y reclamos? }\end{array}$ & $40.00 \%$ & $\begin{array}{c}\text { Medianamente } \\
\text { Satisfactorio }\end{array}$ \\
\hline Seguridad & $\begin{array}{l}\text { ¿Cómo calificaría la } \\
\text { capacidad de los } \\
\text { Profesionales de Salud? }\end{array}$ & $61 \%$ & Satisfactorio \\
\hline
\end{tabular}




\section{CIENCIAMATRIA}

Revista Interdisciplinaria de Humanidades, Educación, Ciencia y Tecnología

Año VII. Vol. VII. N¹2. Enero - Junio. 2021

Hecho el depósito de ley: pp201602FA4721

ISSN-L: 2542-3029; ISSN: 2610-802X

Universidad Nacional Experimental Francisco de Miranda (UNEFM). Santa Ana de Coro. Venezuela

Bella Lourdes Argudo-Pesántez; Cecilia Ivonne Narváez-Zurita

Edwin Joselito Vásquez-Erazo

\begin{tabular}{|c|c|c|c|c|}
\hline & $\begin{array}{l}\text { ¿Considera que las } \\
\text { protecciones de } \\
\text { seguridad del personal } \\
\text { del establecimiento son } \\
\text { apropiadas? }\end{array}$ & $61 \%$ & Satisfactorio & 0 \\
\hline & $\begin{array}{l}\text { ¿Existió comprensión } \\
\text { frente a sus } \\
\text { necesidades y } \\
\text { emociones? }\end{array}$ & $44 \%$ & $\begin{array}{l}\text { Medianamente } \\
\text { Satisfactorio }\end{array}$ & \\
\hline Empatía & $\begin{array}{l}\text { ¿Considera que le } \\
\text { brindaron confianza en } \\
\text { la atención tanto los } \\
\text { Médicos, Enfermeras y } \\
\text { Auxiliares? }\end{array}$ & $60 \%$ & Satisfactorio & 0 \\
\hline
\end{tabular}

Fuente: Los autores.

\section{CONCLUSIONES}

De lo analizado se puede concluir que evaluar la percepción de la satisfacción del cliente externo del Centro Clínico Quirúrgico Ambulatorio Hospital del día de Azogues, es de suma importancia ya que se puede identificar que en general los usuarios se encuentran satisfechos, pero para mejorar las deficiencias encontradas, es necesario implementar estrategias de mejoramiento; así como también se debe realizar evaluaciones periódicas, de los procesos para mejorar los servicios y cubrir las necesidades y expectativas de los usuarios.

Por lo indicado la mayoría de los porcentajes de satisfacción del cliente externo del establecimiento en estudio se encontraron en el rango de satisfacción superior a la media: 


\section{CIENCIAMATRIA}

Revista Interdisciplinaria de Humanidades, Educación, Ciencia y Tecnología

Año VII. Vol. VII. N¹2. Enero - Junio. 2021

Hecho el depósito de ley: pp201602FA4721

ISSN-L: 2542-3029; ISSN: 2610-802X

Universidad Nacional Experimental Francisco de Miranda (UNEFM). Santa Ana de Coro. Venezuela

Bella Lourdes Argudo-Pesántez; Cecilia Ivonne Narváez-Zurita

Edwin Joselito Vásquez-Erazo

1. El aspecto más valorado fue la dimensión de tangibilidad los pacientes están satisfechos con la apariencia de los empleados, las instalaciones físicas (equipamiento e infraestructura).

2. Le sigue entre las más valoradas esta la dimensión de seguridad, en lo que tiene que ver con la capacidad de los Profesionales de Salud y el uso de protecciones de seguridad del personal del establecimiento.

3. Posteriormente el grado la dimensión de empatía, se consideró que la atención que brindan tanto los Médicos, Enfermeras y Auxiliares brindaron confianza a los usuarios.

4. Con La dimensión capacidad de respuesta, los usuarios se encontraron medianamente satisfechos, en la atención del personal de salud para resolver inquietudes y reclamos.

5. En último lugar está la dimensión de fiabilidad, en lo que corresponde a los tiempos de espera para la atención, los usuarios se encuentran insatisfechos, pero existe satisfacción en los horarios establecidos en la institución de salud.

\section{REFERENCIAS CONSULTADAS}

Arbeláez, G., \& Mendoza, P. (2017). Relación entre gestión del director y satisfacción del usuario externo en centros de salud de un distrito del Ecuador. Anales de la Facultad de Medicina, 78(2),

$154-$ 160. https://dx.doi.org/10.15381/anales.v78i2.13197

Blázquez, S., \& Quezada, A. (2014). Satisfaccion de los usuarios hospitalizados en el servicio de medicina interna, con el cuidado de enfermería. Rev Med UV, 14(1):1419.

Cañar, N. d. (2019). Hospital del día de Azogues mejora atención. https://n9.cl/8ynsz

Carrillo, F., Córdova, N., \& Qui, T. (2016). Modelos de satisfacción: fundamentación teórica y criterios de aplicación. INNOVA Research Journal, 1(10), 145-155. https://doi.org/10.33890/innova.v1.n10.2016.64 


\section{CIENCIAMATRIA}

Revista Interdisciplinaria de Humanidades, Educación, Ciencia y Tecnología

Año VII. Vol. VII. N¹2. Enero - Junio. 2021

Hecho el depósito de ley: pp201602FA4721

ISSN-L: 2542-3029; ISSN: 2610-802X

Universidad Nacional Experimental Francisco de Miranda (UNEFM). Santa Ana de Coro. Venezuela

Bella Lourdes Argudo-Pesántez; Cecilia Ivonne Narváez-Zurita

Edwin Joselito Vásquez-Erazo

Fariño, J., Cercado, A., Loreti, E., \& Valle, J. (2018). Satisfacción de los usuarios y la calidad de atención que se brinda en las unidades operativas de atención primaria de salud. Revista Espacios, 39(32), 22.

Godínez, F., Godínez, F., Ariza, F., Reducindo, M., Cuevas, A., \& Cabañas, E. (2014). Nivel de satisfacción de usuarios de consulta externa en un hospital público. Psicología y Salud, 24(1), 97-107.

González-Arias, M, Frías-Jiménez, R, \& Gómez-Figueroa, O. (2016). Análisis de la calidad percibida por el cliente en la actividad hotelera. Ingeniería Industrial, 37(3), 253-265.

Guadalupe-Rivera, F., Suárez-Lima, G. J., Guerrero-Lapo, G. E., \& Yancha-Moreta, C. A. (2019). Satisfacción de los usuarios y la calidad de atención que se brinda en el área de emergencia del hospital general Instituto Ecuatoriano de Seguridad Social Milagro; 2018. Ciencia Digital, 3(3), 162-177. https://doi.org/10.33262/cienciadigital.v3i3.621

Huerta-Medina, E. (2015). Nivel De Satisfacción De Los Usuarios Atendidos En El Tópico De Medicina Del Servicio De Emergencia Del Hospital Nacional Arzobispo Loayza, Lima, Enero Del 2015. https://n9.cl/e15jt

Ibarra, L., \& Casas, E. (2015). Aplicación del modelo Servperf en los centros de atención Telcel, Hermosillo: una medición de la calidad en el servicio. Contaduría y administración, 60(1), 229-260.

Infantes-Gómez, F. (2017). Calidad de atención y grado de satisfacción de los usuarios de consulta externa del Centro de Atención de Medicina Complementaria del Hospital III Iquitos-2016. Revista Peruana de Medicina Integrativa, 2(2), 133-139. http://dx.doi.org/10.26722/rpmi.2017.22.55

Juran, J. (1988). Juran y la planificación de la calidad. https://n9.cl/uobou

Matsumoto Nishizawa, Reina. (2014). Desarrollo del Modelo Servqual para la medición de la calidad del servicio en la empresa de publicidad Ayuda Experto. Revista Perspectivas, (34), 181-209.

Méndez-Matovelle, A., Quevedo-Barros, M., Carangui-Velecela, P., \& Jácome-Ortega, M. (2020). Gestión de compras como estrategia competitiva de las organizaciones. Revista Arbitrada Interdisciplinaria Koinonía, 5(3), 97-124. 


\section{CIENCIAMATRIA}

Revista Interdisciplinaria de Humanidades, Educación, Ciencia y Tecnología

Año VII. Vol. VII. N¹2. Enero - Junio. 2021

Hecho el depósito de ley: pp201602FA4721

ISSN-L: 2542-3029; ISSN: 2610-802X

Universidad Nacional Experimental Francisco de Miranda (UNEFM). Santa Ana de Coro. Venezuela

Bella Lourdes Argudo-Pesántez; Cecilia Ivonne Narváez-Zurita

Edwin Joselito Vásquez-Erazo

Mora-Contreras, C. (2011). La calidad del servicio y la satisfacción del consumidor. Revista Brasileira de Marketing, 10(2),146-162.

Numpaque-Pacabaque, A, \& Rocha-Buelvas, A. (2016). Modelos SERVQUAL y SERVQHOS para la evaluación de calidad de los servicios de salud. Revista de la Facultad de 720. https://dx.doi.org/10.15446/revfacmed.v64n4.54839

Pitaluga, R. (2015). Comentarios sobre el modelo de Noriaki Kano. https://n9.cl/6zvap

Ramón-Ochoa, M. del R., Erazo-Álvarez, J. C., Narváez-Zurita, C. I., \& MatovelleRomo, M. M. (2019). Gestión del capital intelectual en las cooperativas de ahorro y crédito de la ciudad de Loja aplicando el modelo Servqual. Visionario Digital, 3(2.2), 284-308. https://doi.org/10.33262/visionariodigital.v3i2.2.644

Ríos-Cáceres, A., Barreto-Galeano, A., \& Martínez-Duque, A. (2016). Percepción de la satisfacción en la atención en salud en usuarios del servicio de consulta externa, de los Hospitales San Antonio de Chía y Santa Rosa de Tenjo, Marzo de 2016. Parte 1 Municipio de Chía; Hospital San Antonio de Chía, Marzo de 2016 Parte 2 Municipio De Tenjo; Hospital Santa Rosa De Tenjo, Marzo Del 2016. https://repository.udca.edu.co/handle/11158/482

Romero, D. (2017). Informe de Rendición de Cuentas Centro Clínico Quirúrgico Ambulatorio Hospital del Día Azogues. https://n9.cl/xgrt

Sánchez-Lunavictoria, J. C., Navas Zamora, S. A., Chávez Rojas, J. I., Fernanda Miranda, M., \& Morocho Quisnancela, J. C. (2019). Implementación del modelo Servperf como herramienta para el diagnóstico y propuesta de mejoras en la prestación del Servicio. Caso: Instituto Ecuatoriano de Seguridad Social Agencia Riobamba. Ciencia Digital, 3(2),

475-483. https://doi.org/10.33262/cienciadigital.v3i2.445

Sandoval, D., Díaz, K., Vasquez, M., Vásquez, P., Vera, L., \& Yacarini, A. (2018). Nivel de satisfacción de usuarios hospitalizados en servicio de cirugía del hospital regional Lambayeque - 2018. Revista Experiencia En Medicina Del Hospital Regional Lambayeque, 4(4), 138 - 142.

Terán, G., Mora, E., Gutiérrez, M., Maldonado, S., Delgado, W., \& A. F. (2017). La Gestión de la Innovación en los Servicios de Salud Pública. Revista Cubana de Investigaciones Biomédicas, 36(3). 


\section{CIENCIAMATRIA}

Revista Interdisciplinaria de Humanidades, Educación, Ciencia y Tecnología

Año VII. Vol. VII. N¹2. Enero - Junio. 2021

Hecho el depósito de ley: pp201602FA4721

ISSN-L: 2542-3029; ISSN: 2610-802X

Universidad Nacional Experimental Francisco de Miranda (UNEFM). Santa Ana de Coro. Venezuela

Bella Lourdes Argudo-Pesántez; Cecilia Ivonne Narváez-Zurita

Edwin Joselito Vásquez-Erazo

Terán, J. (2020). Clima laboral y calidad de atención en salud en el centro de salud tipo a IESS. Cotacachi. http://repositorio.utn.edu.ec/handle/123456789/10345

Valencia, E. S. (2015). Aplicación del modelo SERVQUAL para la medición de la calidad del servicio de mantenimiento y limpieza de establecimientos educativos en la empresa SYRY. https://dspace.ups.edu.ec/handle/123456789/10269

(C2021 por los autores. Este artículo es de acceso abierto y distribuido según los términos y condiciones de la licencia Creative Commons Atribución-NoComercial-Compartirlgual 4.0 Internacional (CC BY-NC-SA 4.0)

(https://creativecommons.org/licenses/by-nc-sa/4.0/). 